INT. J. CONTROL, 1990, VOL. 51, NO. 1, 11-31

\title{
Control and stabilization of a flexible beam attached to a rigid body
}

\section{Ö. MORGÜL†}

We consider a flexible spacecraft modelled as a rigid body rotating in inertial space; a light flexible beam is clamped to the rigid body at one end and is free at the other. The equations of motion are obtained by using free-body diagrams. It is shown that suitable boundary controls applied to the free end of the beam and a control torque applied to the rigid body stabilize the system. The proof is obtained by using the energy of the system as a Lyapunov functional.

\section{Introduction}

Many mechanical systems, such as spacecraft with flexible appendages, consist of coupled elastic and rigid parts. In such systems, if a good performance of the overall system is desired, the dynamic effect of elastic members becomes important. Thus over the last decade there has been growing interest in developing new methods for the design, dynamics and control of systems that have elastic parts (see e.g. Balas (1982) and references therein).

Consider a system that has rigid and elastic members. The motion of the elastic members is usually described by a set of partial differential equations with appropriate boundary conditions. Since the motion of the rigid parts is governed by a set of nonlinear ordinary differential equations and the rigid members are coupled with elastic members, the overall equations of motion generally form a set of coupled non-linear partial and ordinary differential equations. These equations can be obtained using standard methods in mechanics (see e.g. Goldstein 1980).

After having obtained the equations of motion, the most commonly used approach is to consider only finitely many modes of the elastic parts - this is called 'modal analysis'. This approach reduces the original equations to a set of coupled nonlinear ordinary differential equations. However, the establishment of a control law for this reduced set of equations does not always guarantee that the same control law will work on the original set of equations (for example, one might encounter so-called 'spillover' problems, Balas 1978). Also it should be noted that the actual number of modes of an elastic system is, in theory, infinite and the number of modes that should be retained is not known a priori.

Recently Biswas and Ahmed (1986) used a Lyapunov-type approach to prove the stability of a rigid spacecraft with an elastic beam attached to it under appropriate forces and torques appiied to the beam and the rigid spacecraft. Their proposed control laws contain distributed forces applied to the beam that are proportional to the beam deflection velocities. Implementation of such control laws might not be easy.

In recent years, boundary control of elastic systems (i.e. controls applied to the boundaries of elastic systems) has become an important research area. This idea was first applied to systems governed by a wave equation, e.g. strings (Chen 1979), and

Received 20 April 1989.

† Department of Electrical and Electronics Engineering, Bilkent University, PK8, Maltepe 06572, Ankara, Turkey. 
recently extended to the beam equations. In particular, Chen et al. (1987) proved that in a cantilever beam a single actuator applied at the free end of the beam is sufficient to uniformly stabilize the beam deflections. This approach has recently been applied to the rigid-body-elastic-beam configuration (Desoer and Morgul 1988).

In this paper we consider the motion of a rigid body with a beam clamped to it, the other end of the beam being free. The rigid body is assumed to be rotating with its centre of mass fixed in an inertial frame. After having obtained the equations of motion, we define the rest state of the system. We then state the control problem, which is, if the system is perturbed from the rest state, to find appropriate control laws that drive the system to the rest state. We propose two different control laws, each of which consist of appropriate boundary force and moment controls applied to the beam at its free end and a torque control applied to the rigid body. We then show that the proposed control laws solve the control problem posed above; that is, the rigid body angular velocity and the beam deflections decay to the rest state.

In $\S 2$ we explain the configuration under consideration and derive the equations of motion using free-body diagrams. We then state the control problem and propose some feedback laws. In $\$ 3-5$ we show that the proposed control laws solve the control problem posed above.

\section{Notation}

Boldface letters $\mathbf{r}, \mathbf{n}$ etc. denote vectors in $\mathbb{R}^{3}$, unless stated otherwise; $f_{x}, f_{\mathbf{t}}$ etc. denote $\partial f / \partial x, \partial f / \partial t$ etc. $\times$ denotes the standard cross-product in $\mathbb{R}^{3}$ and $\langle$,$\rangle denotes$ the standard inner product in $\mathbb{R}^{3}$.

\section{The configuration}

\subsection{Equations of motion}

We consider the configuration shown in the Figure, where the rigid body is drawn as a square and $P$ is a point on the beam. $\left(O, \mathbf{e}_{1}, \mathbf{e}_{2}, \mathbf{e}_{3}\right)$ denotes a right-handed orthonormal inertial frame, which will be referred to as $\mathbf{N},\left(O, \mathbf{D}_{1}, \mathbf{D}_{2}, \mathbf{D}_{3}\right)$ denotes a right-handed orthonormal frame fixed in the rigid body, which will be referred as $\mathbf{B}$, where $O$ is also the centre of mass of the rigid body and $\mathbf{D}_{1}, \mathbf{D}_{2}, \mathbf{D}_{3}$ are along the

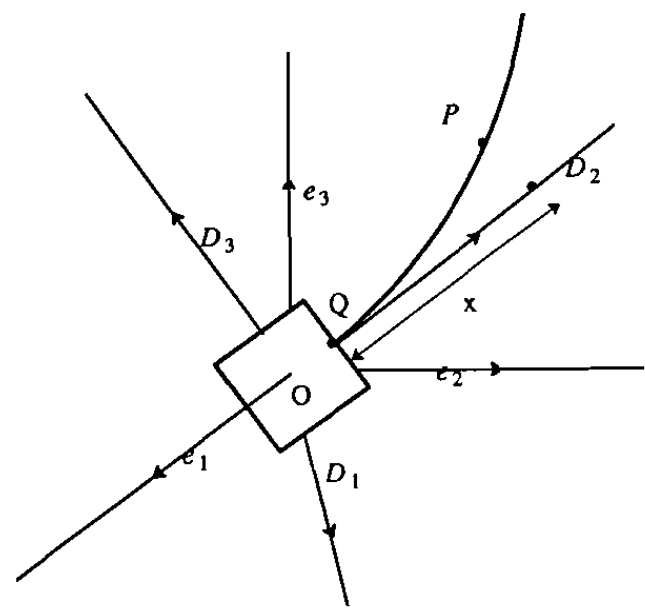

Rigid body with flexible beam. 
principal axes of inertia of the rigid body. One end of the beam is clamped to the rigid body at the point $Q$ along the $\mathbf{D}_{2}$ axis and the other end is free. Let $L$ be the length of the beam. We assume that the mass of the rigid body is much larger than the mass of the beam, so the centre of mass of the rigid body is approximately the centre of mass of the whole configuration. So the point $O$ is fixed in inertial space throughout the motion of the whole configuration, and the rigid body may rotate arbitrarily in the inertial space.

The beam is initially straight, along the $\mathbf{D}_{2}$ axis. Let $P$ be a typical beam element whose distance from $Q$ in the undeformed configuration is $x$, and let $u_{1}$ and $u_{3}$ be the displacements of $P$ along the $\mathbf{D}_{1}$ and $\mathbf{D}_{3}$ axes, respectively. We assume that the beam is inextensible; that is, the beam deflection $u_{2}$ along the $\mathbf{D}_{2}$ axis is identically zero. Let $\mathbf{r}(x, t)=\mathbf{O P}$ be the position vector of $P$. We assume that the beam is homogeneous with uniform cross-sections.

We define the contact force $\mathbf{n}(x, t)$ and the contact moment $\mathbf{m}(x, t)$ at the beam cross-sections as follows. Consider a beam cross-section $C_{x}$ at $x$. The effect of the part of the beam that lies on the $(x, L]$ segment of the beam on the part that lies on the $[0, x]$ segment is equivalent to a force applied to the cross-section $C_{x}$, which is called the contact force $\mathrm{n}(x, t)$, and a moment applied to the cross-section $C_{x}$, which is called the contact moment $\mathbf{m}(x, t)$. For further information see Antman (1972).

Neglecting gravitation, surface loads and rotatory inertia of the beam crosssections, we obtain the following equations describing the motion of the whole configuration for $t \geqslant 0$ :

$$
\begin{gathered}
\frac{\partial \mathbf{n}}{\partial x}=\frac{\partial^{2} \mathbf{r}}{\partial t^{2}} \quad(0<x<L) \\
\frac{\partial \mathbf{m}}{\partial x}+\frac{\partial \mathbf{r}}{\partial x} \times \mathbf{n}=0 \quad(0<x<L) \\
\mathbf{I}_{\mathbf{R}} \cdot \dot{\boldsymbol{\omega}}+\boldsymbol{\omega} \times \mathrm{I}_{R} \cdot \boldsymbol{\omega}=\mathbf{r}(0, t) \times \mathbf{n}(0, t)+\mathbf{m}(0, t)+\mathbf{N}_{\mathbf{c}}(t)
\end{gathered}
$$

where $\mathbf{n}(x, t)$ and $\mathbf{m}(x, t)$ are the contact force and the contact moment respectively, $\lambda$ is the mass per unit length of the beam (which is constant by assumption), $L$ is the length of the beam, $I_{R}$ is the inertia tensor of the rigid body (which is diagonal), $\omega$ is the angular velocity of the rigid body with respect to the inertial frame $\mathbf{N}$, and $\mathbf{N}_{c}(t)$ is the control torque applied to the rigid body (see e.g. Antman 1972).

Equations (2.1) and (2.2) state the balance of forces and the balance of moments at the beam cross-sections, and (2.3) is the rigid-body angular-momentum equation. Note that the first two terms on the right-hand side of (2.3) represent the torque applied by the beam to the rigid body.

\section{Remark 2.1}

Let $\mathbf{r}: \mathbb{R} \rightarrow \mathbb{R}^{3}$ denote a vector-valued function of time, typically $\mathbf{r}(t)$ is the position of a particle. Let $\mathbf{r}^{N}=\left(r_{1}^{N}, r_{2}^{N}, r_{3}^{N}\right)^{\mathrm{T}}$ and $\mathbf{r}^{B}=\left(r_{1}^{B}, r_{2}^{B}, r_{3}^{B}\right)^{\mathrm{T}}$ denote the components of $\mathbf{r}$ in the right-handed orthonormal frame $\mathbf{N}$ given by $\left(O, \mathbf{e}_{1}, \mathbf{e}_{2}, \mathbf{e}_{3}\right)$ and in the righthanded orthonormal frame $\mathbf{B}$ given by $\left(O, \mathbf{D}_{1}, \mathbf{D}_{2}, \mathbf{D}_{3}\right)$ respectively. Let $\boldsymbol{\omega}$ denote the angular velocity of the frame $\mathbf{B}$ with respect to the frame $\mathbf{N}$. Then we have the following (see Kane and Levinson 1985):

$$
\sum_{i=1}^{i=3} \frac{d r_{i}^{N}}{d t} \mathbf{e}_{i}=\sum_{i=1}^{i=3} \frac{d r_{i}^{B}}{d t} \mathbf{D}_{i}+\boldsymbol{\omega} \times \mathbf{r}
$$


If we define

$$
\left(\frac{d \mathbf{r}}{d t}\right)_{N}=\sum_{i=1}^{i=3} \frac{d r_{i}^{N}}{d t} \mathbf{e}_{i}, \quad\left(\frac{d \mathbf{r}}{d t}\right)_{B}=\sum_{i=1}^{i=3} \frac{d r_{i}^{B}}{d t} \mathbf{D}_{i}
$$

then we obtain the following equation (see e.g. Goldstein 1980):

$$
\left(\frac{d \mathbf{r}}{d r}\right)_{N}=\left(\frac{d \mathbf{r}}{d t}\right)_{B}+\boldsymbol{\omega} \times \mathbf{r}
$$

We use the Euler-Bernoulli beam model to give the component form of the contract force $\mathbf{n}$ and the contact moment $\mathbf{m}$ in terms of the beam deflections $u_{1}$ and $u_{3}$. For more details see Meirovitch (1967). Assuming that the beam is inextensible, neglecting the torsion and neglecting the higher-order terms, we express the contact force $\mathbf{n}$, the contact moment $\mathbf{m}$ and the position vector $\mathbf{r}$ in terms of $u_{1}$ and $u_{3}$ as follows: for $0 \leqslant x \leqslant L, t \geqslant 0$

$$
\begin{aligned}
\mathbf{m} & =m_{1} \mathbf{D}_{1}+m_{2} \mathbf{D}_{2}+m_{3} \mathbf{D}_{3}, \quad \mathbf{n}=n_{1} \mathbf{D}_{1}+n_{2} \mathbf{D}_{2}+n_{3} \mathbf{D}_{3} \\
m_{1} & =E l_{3} u_{3 x x}, \quad n_{3}=-E l_{3} u_{3 x x x} \\
m_{3} & =-E I_{1} u_{1 x x}, \quad n_{1}=-E I_{1} u_{1 x x x x} \\
r & =u_{1} \mathbf{D}_{1}+(b+x) \mathbf{D}_{2}+u_{3} \mathbf{D}_{3}
\end{aligned}
$$

where $E I_{1}$ and $E I_{3}$ are the flexural rigidities of the beam deflections along the axes $\mathbf{D}_{1}$ and $\mathbf{D}_{3}$ respectively and $b$ is the distance between the points $O$ and $Q$.

Since we have neglected the axial and torsional vibrations of the beam, the axial component $n_{2}$ of the contact force $n$ and the torsion component $m_{2}$ of the contact moment $\mathbf{m}$ are not determinable from the constitutive equations (see Posbergh 1988). Once the beam deflections $u_{1}$ and $u_{3}$ have been found, the $\mathbf{D}_{2}$ components of $(2.1)$ and (2.2) may be used to find $n_{2}$ and $m_{2}$.

Since the beam is clamped to the rigid body at the point $Q$, we have (see the Figure)

$$
u_{i}(0, t)=u_{i x}(0, t)=0, \quad t \geqslant 0, \quad i=1,3
$$

The rest state of the system is, by definition,

$$
\begin{aligned}
\omega & =0 \\
u_{1}(x) & =u_{3}(x)=0, \quad 0 \leqslant x \leqslant L \\
u_{1 t}(x) & =u_{3 t}(x)=0, \quad 0 \leqslant x \leqslant L
\end{aligned}
$$

We now state our stabilization problem.

\section{Stabilization problem}

If the system given by $(2.1)-(2.9)$ is perturbed from the rest state defined by (2.10) then find an appropriate control law that drives the system to the rest state.

\subsection{Proposed control laws}

We propose two stabilizing control laws. Each law consists of appropriate forces and torques applied to the beam at the free end and a torque applied to the rigid body. We note that these two sets differ in the torque applied to the rigid body. 


\subsubsection{Control law based on cancellation}

This control scheme applies a force $\mathbf{n}(L, t)$ and a torque $\mathbf{m}(L, t)$ at the free end of the beam and a torque $\mathbf{N}_{\mathrm{c}}(t)$ to the rigid body. They are specified as follows: we choose $\alpha_{i}>0, \beta_{i}>0$, and a $3 \times 3$ positive-definite constant matrix $\mathbf{K}$ (which can be chosen diagonal); then for all $t \geqslant 0, i=1,3$, we require the following equations:

$$
\begin{aligned}
n_{i}(L, t)+\alpha_{i} u_{i t}(L, t) & =0 \\
m_{i}(L, t)+\beta_{i} u_{i x t}(L, t) & =0 \\
\mathbf{N}_{\mathrm{c}}(t) & =-\mathbf{r}(0, t) \times \mathbf{n}(0, t)-\mathbf{m}(0, t)-\mathbf{K} \cdot \boldsymbol{\omega}(t)
\end{aligned}
$$

Equation (2.11) (respectively (2.12)) represents a transverse force (respectively torque) applied at the free end of the beam in the direction (respectively around) the axis $\mathbf{D}_{i}$ whose magnitude is proportional to and whose sign is opposite to the endpoint deflection velocity $u_{i t}(L, t)$ (respectively end-point deflection angular velocity $\left.u_{i x i}(L, t)\right)$ of the beam along the direction of the $\mathbf{D}_{i}$ axis, for $i=1,3$. Also note that to apply the control laws given by $(2.11)-(2.13 a)$, the end-point deflection velocities $u_{i t}(L, t)$, the end-point deflection angular velocities $u_{i x}(L, t)$, the rigid-body angularvelocity vector $\omega(t)$ and the moment applied by the beam to the rigid body must be measured. This moment consists of the effect of the contact force $n(0, t)$ and the contact moment $\mathbf{m}(0, t)$ at the clamped end. Both can be measured by using strain rosettes and strain gauges respectively (J. Anagnost, 1988, personal communication).

The control law $(2.13 a)$ cancels the effect of the beam on the rigid body. To see this, substitute $(2.13 a)$ into (2.3), then (2.3) becomes a set of non-linear ordinary differential equations. Then substitute the solution $\omega(t)$ of (2.3) into the beam equation (2.1). Now the latter becomes a set of linear partial differential equations.

Equation $(2.13 a)$ is reminiscent of a 'computed-torque' type control law in robotics (Paul 1981). When substituted into $(2.3),(2.13 a)$ cancels the effect of the beam on the rigid body. This type of control law has recently been applied to attitude control of flexible spacecraft (J. Anagnost, 1988, personal communication).

\subsubsection{Natural control law}

This control scheme applies the same boundary force $\mathbf{n}(L, t)$ and moment $\mathbf{m}(L, t)$ as specified by (2.11) and (2.12) respectively, but the torque applied to the rigid body is given by

$$
\mathbf{N}_{\mathrm{c}}(t)=-\mathbf{r}(L, t) \times \mathbf{n}(L, t)-\mathbf{m}(L, t)-\mathbf{K} \cdot \boldsymbol{\omega}(t)
$$

where $\mathbf{K}$ is a $3 \times 3$ positive-definite constant matrix.

This control scheme is 'natural' in the sense that it enables one to choose the total energy of the whole configuration as a Lyapunov function to study the stability of the system.

Unlike the control law $(2.13 a)$, when $(2.13 b)$ is substituted into $(2.3)$, it does not cancel the effect of the beam on the rigid body. As a result of this, (2.1)-(2.9), together with the control laws $(2.11),(2.12)$ and $(2.13 \mathrm{~b})$ form a set of non-linear ordinary and partial differential equations. The control law $(2.11),(2.12),(2.13 b)$ requires that the end-point deflections $u_{i}(L, t)$, the end-point deflection velocities $u_{i t}(L, t)$, the end-point deflection angular velocities $u_{i x t}(L, t)$ and the rigid-body angular-velocity vector $\omega(t)$ be measured. The first three could be measured by optical means and the latter by gyros. 
Throughout our analysis, the initial conditions $u_{i}(x, 0)$ and $u_{i r}(x, 0)$ are assumed to be sufficiently differentiable (i.e. $C^{2}$ in $t$ and $C^{4}$ in $x$ ) and compatible with the boundary conditions (2.9), (2.11) and (2.12) for $i=1,3$.

\section{Stability results for the control law based on cancellation}

After substituting ( $2.13 a)$ into (2.3) we obtain the following rigid-body equation:

$$
I_{R} \cdot \dot{\omega}+\omega \times I_{R} \cdot \omega=-K \cdot \omega
$$

\section{Proposition 3.1}

Consider (3.1). There exist a $c>0$ and an $\alpha>0$ such that for all initial conditions $\omega(0) \in \mathbb{R}^{3}$ the solution $\omega(t)$ of $(2.1)$ satisfies

$$
\langle\omega(t), \omega(t)\rangle \leqslant c e^{-\alpha t}\langle\omega(0), \omega(0)\rangle \text { for all } t \geqslant 0
$$

Proof

Consider the following 'energy function' for the rigid body:

$$
E_{\mathbf{R}}(t)=\frac{1}{2}\left\langle\boldsymbol{\omega}(t), \mathbf{I}_{\mathbf{R}} \cdot \boldsymbol{\omega}(t)\right\rangle
$$

$E_{\mathrm{R}}(t)$ is the rotational kinetic energy of the rigid body with respect to the inertial frame $\mathbf{N}$. Also note that, since $I_{\mathrm{R}}=\operatorname{diag}\left(I_{1}, I_{2}, I_{3}\right)$, we have

$$
I_{\min }\langle\boldsymbol{\omega}, \boldsymbol{\omega}\rangle \leqslant 2 E_{\mathrm{R}} \leqslant I_{\max }\langle\boldsymbol{\omega}, \boldsymbol{\omega}\rangle \text { for all } \boldsymbol{\omega} \in \mathbb{R}^{3}
$$

where $I_{\min }=\min \left(I_{1}, I_{2}, I_{3}\right)$ and $I_{\max }=\max \left(I_{1}, I_{2}, I_{3}\right)$. Differentiating (3.3) and using (3.1), we obtain

$$
\begin{aligned}
\dot{E}_{\mathbf{R}}(t) & =\left\langle\boldsymbol{\omega}, \mathbf{I}_{\mathbf{R}} \cdot \dot{\boldsymbol{\omega}}\right\rangle \\
& =-\left\langle\boldsymbol{\omega}, \boldsymbol{\omega} \times \mathbf{I}_{\mathbf{R}} \cdot \boldsymbol{\omega}\right\rangle-\langle\boldsymbol{\omega}, \mathbf{K} \cdot \boldsymbol{\omega}\rangle \\
& =-\langle\boldsymbol{\omega}, \mathbf{K} \cdot \boldsymbol{\omega}\rangle
\end{aligned}
$$

But since $\mathbf{K}$ is positive-definite, there exist positive non-zero constants $\lambda_{1}$ and $\lambda_{2}$, which may be taken as the minimum and the maximum eigenvalues of $\frac{1}{2}\left(K+K^{T}\right)$ respectively such that the following holds:

$$
\lambda_{1}\langle\boldsymbol{\omega}, \boldsymbol{\omega}\rangle \leqslant\langle\boldsymbol{\omega}, \mathbf{K} \cdot \boldsymbol{\omega}\rangle \leqslant \lambda_{2}\langle\boldsymbol{\omega}, \boldsymbol{\omega}\rangle
$$

Using (3.4)-(3.6), we obtain (3.2) where

$$
c=\frac{\max \left(I_{1}, I_{2}, I_{3}\right)}{\min \left(I_{1}, I_{2}, I_{3}\right)}, \quad \alpha=\frac{2 \lambda_{1}}{\max \left(I_{1}, I_{2}, I_{3}\right)}
$$

Next, we obtain the component form of (2.1). After applying (2.4) twice, we obtain

$$
\left(\frac{d^{2} \mathbf{r}}{d t^{2}}\right)_{N}=\left(\frac{d^{2} \mathbf{r}}{d t^{2}}\right)_{B}+\dot{\omega} \times \mathbf{r}+2 \boldsymbol{\omega} \times\left(\frac{d \mathbf{r}}{d t}\right)_{B}+\boldsymbol{\omega} \times(\boldsymbol{\omega} \times \mathbf{r})
$$

Using (3.7) in (2.1) - (2.10), we obtain the following equations governing the motion of transverse beam deflections in the $\mathbf{D}_{1}$ and $\mathbf{D}_{3}$ directions:

$$
\begin{aligned}
E I_{1} u_{1 x x x x}+\lambda u_{1 t t}+2 i \omega_{2} u_{3 t}+\lambda\left(\dot{\omega}_{2}+\omega_{1} \omega_{3}\right) u_{3} & \\
& -\lambda\left(\omega_{2}^{2}+\omega_{3}^{2}\right) u_{1}-\lambda\left(\dot{\omega}_{3}-\omega_{1} \omega_{2}\right)(b+x)=0, \quad 0<x<L, \quad t \geqslant 0
\end{aligned}
$$




$$
\begin{aligned}
E I_{3} u_{3 x x x x} & +\lambda u_{3 t}-2 \lambda \omega_{2} u_{1 t}-\lambda\left(\dot{\omega}_{2}-\omega_{1} \omega_{3}\right) u_{1} \\
& -\lambda\left(\omega_{1}^{2}+\omega_{2}^{2}\right) u_{3}+\lambda\left(\dot{\omega}_{1}+\omega_{2} \omega_{3}\right)(b+x)=0, \quad 0<x<L, \quad t \geqslant 0
\end{aligned}
$$

Equations (3.8) and (3.9) can be rewritten in the following state-space form:

$$
\begin{aligned}
& \frac{d}{d t}\left[\begin{array}{c}
u_{1} \\
u_{1 t} \\
u_{3} \\
u_{3 t}
\end{array}\right]=\left[\begin{array}{cccc}
0 & 1 & 0 & 0 \\
-\frac{E I_{1}}{\lambda} \frac{\partial^{4}}{\partial x^{4}} & 0 & 0 & 0 \\
0 & 0 & 0 & 1 \\
0 & 0 & -\frac{E I_{3}}{\lambda} \frac{\partial^{4}}{\partial x^{4}} & 0
\end{array}\right]\left[\begin{array}{c}
u_{1} \\
u_{1 t} \\
u_{3} \\
u_{3 t}
\end{array}\right] \\
& +\left[\begin{array}{cccc}
0 & 0 & 0 & 0 \\
\omega_{2}^{2}+\omega_{3}^{2} & 0 & -\left(\dot{\omega}_{2}+\omega_{1} \omega_{3}\right) & -2 \omega_{2} \\
0 & 0 & 0 & 0 \\
\dot{\omega}_{2}-\omega_{1} \omega_{3} & 2 \omega_{2} & \omega_{1}^{2}+\omega_{2}^{2} & 0
\end{array}\right]\left[\begin{array}{c}
u_{1} \\
u_{1 t} \\
u_{3} \\
u_{3 t}
\end{array}\right] \\
& +\left[\begin{array}{c}
0 \\
\left(\dot{\omega}_{3}-\omega_{1} \omega_{2}\right)(b+x) \\
0 \\
-\left(\dot{\omega}_{1}+\omega_{2} \omega_{3}\right)(b+x)
\end{array}\right]
\end{aligned}
$$

whose solutions evolve in the following function space:

$$
H=\left\{\left(u_{1}, u_{1 t}, u_{3}, u_{3 t}\right) \mid u_{1} \in \mathbf{H}_{0}^{2}, u_{3} \in \mathbf{H}_{0}^{2}, u_{1 t} \in \mathbf{L}^{2}, u_{3 t} \in \mathbf{L}^{2}\right\}
$$

where the function spaces $\mathbf{L}^{2}, \mathbf{H}^{k}$ and $\mathbf{H}_{0}^{k}$ are defined by

$$
\begin{aligned}
& \mathbf{L}^{2}=\left\{f:[0, L] \rightarrow \mathbb{R} \mid \int_{0}^{L} f^{2} d x<\infty\right\} \\
& \mathbf{H}^{k}=\left\{f \in \mathbf{L}^{2} \mid f^{i} \in \mathbf{L}^{2}, i=1, \ldots, k\right\} \\
& \mathbf{H}_{0}^{k}=\left\{f \in \mathbf{H}^{k} \mid f(0)=f^{1}(0)=0\right\}
\end{aligned}
$$

In $H$ we define the following inner product, which is called the 'energy' inner product:

$$
\begin{aligned}
\langle z, \hat{\mathrm{z}}\rangle_{E}:= & \int_{0}^{L}\left(E I_{1} u_{1 x x} \hat{u}_{1 x x}+E I_{3} v_{1 x x} \hat{v}_{1 x x}\right) d x \\
& +\int_{0}^{L} \lambda\left(u_{2} \hat{u}_{2}+v_{2} \hat{v}_{2}\right) d x \quad \text { for all } z, \hat{z} \in H
\end{aligned}
$$

Note that (3.12) induces a norm on $H$, which is called the 'energy norm'. This norm is equivalent to a standard 'Sobolev' type norm, which makes $H$ into a Hilbert space (for more details, see Pazy 1983, Chen et al. 1987).

To put (3.10) into an abstract equation form, we define the following operators 
$A: H \rightarrow H, B: \mathbb{R}^{+} \times H \rightarrow H$ and a function $f: \mathbb{R}^{+} \rightarrow \mathrm{H}:$

$$
\begin{aligned}
A & =\left[\begin{array}{cccc}
0 & 1 & 0 & 0 \\
-\frac{E I_{1}}{\lambda} \frac{\partial^{4}}{\partial x^{4}} & 0 & 0 & 0 \\
0 & 0 & 0 & 1 \\
0 & 0 & -\frac{E I_{3}}{\lambda} \frac{\partial^{4}}{\partial x^{4}} & 0
\end{array}\right] \\
B(t) & =\left[\begin{array}{cccc}
\omega_{2}^{2}+\omega_{3}^{2} & 0 & -\left(\dot{\omega}_{2}+\omega_{1} \omega_{3}\right) & -2 \omega_{2} \\
0 & 0 & 0 & 0 \\
\dot{\omega}_{2}-\omega_{1} \omega_{3} & 2 \omega_{2} & \omega_{1}^{2}+\omega_{3}^{2} & 0 \\
0 & 0
\end{array}\right] \\
f(t) & =\left[\begin{array}{c}
\left(\dot{\omega}_{3}-\omega_{1} \omega_{2}\right)(b+x) \\
0 \\
-\left(\dot{\omega}_{1}+\omega_{2} \omega_{3}\right)(b+x)
\end{array}\right]
\end{aligned}
$$

\section{Remark 3.1}

$A$ is an unbounded linear operator on $H . B(t)$ is bounded on $\mathbb{R}^{+}$. Since $\omega(t)$ and $\dot{\omega}(t)$ are exponentially decaying functions of $t$ (see Proposition 3.1 and (3.1)), so is $\|B(t)\|$, where the norm used here is the norm induced by the energy inner product given by (3.12).

Using the above definitions, (3.10) can be put into the following abstract form:

$$
\frac{d z}{d t}=A z+B(t) z+f(t), \quad z(0)=z_{0} \in H
$$

where $z=\left[\begin{array}{llll}u_{1} & u_{11} & u_{3} & u_{3 t}\end{array}\right]^{\mathrm{T}}$. The domain $D(A)$ of the operator $A$ is defined as follows:

$$
\begin{aligned}
D(A)=\left\{\left(u_{1}, u_{1 t}, u_{3}, u_{3} t\right): u_{1} \in H_{0}^{4}, u_{3} \in H_{0}^{4}, u_{1 t} \in H_{0}^{2}, u_{3 t} \in H_{0}^{2}\right. \\
-E I_{1} u_{1 x x x}(L)+\alpha_{1} u_{1 t}(L)=0, \\
E I_{1} u_{1 x x}(L)+\beta_{1} u_{1 x t}(L)=0, \\
-E I_{3} u_{3 x x x}(L)+\alpha_{3} u_{3 t}(L)=0, \\
\left.E I_{3} u_{3 x x}(L)+\beta_{3} u_{3 x t}(L)=0\right\} .
\end{aligned}
$$

It is easy to show that $D(A)$ is dense in $H$ (see Chen et al. 1987).

Next, we state the existence and uniqueness theorem of the solutions of $(2.16)$.

\section{Fact 3.1}

Consider (3.16) with $A, B$ and $f$ defined in (3.13)- $-(3.15)$. Then 
(i) The operator $A$ generates an exponentially decaying $C_{0}$ semigroup $T(t)$ in $H$; that is, there exist an $M>0$ and a $\delta>0$ such that

$$
\|T(t)\| \leqslant M e^{-\delta t} \text { for all } t \geqslant 0
$$

where for all $t \geqslant 0, T(t)$ are bounded linear maps in $H$;

(ii) for all $z_{0} \in D(A),(3.16)$ has unique classical solution, defined for all $t \geqslant 0$;

(iii) in terms of $T(t)$, that solution $z(t)$ of (3.16) may be written as:

$$
z(t)=T(t) z_{0}+\int_{0}^{t} T(t-s) B(s) z(s) d s+\int_{0}^{t} T(t-s) f(s) d s \text { for all } t \geqslant 0
$$

Proof

(i) Because of the block-diagonal form of $A$, this is an easy extension of Theorem 3.1 of Chen et al. (1987).

(ii) Since $B(t)$ is globally Lipschitz on $H$ and $\|B(t)\|$ is exponentially decaying, owing to Proposition 3.1 (see also Remark 3.1), it follows that $A+B(t)$ defines a unique, globally defined semigroup on $H$ (see e.g. Marsden 1983, Pazy 1983). Since $f \in L^{1}[R, H]$ and is a $C^{\infty}$ function of $t$ (see (3.15)), by standard theorems on non-homogeneous linear partial differential equations (see e.g. Pazy 1983, pp. 105-110), it follows that (3.16) has a unique solution defined for all $t \geqslant 0$.

(iii) That the solution may be given as (3.19) can be verified by substitution, using $d T / d t=A T$.

Next, we prove the exponential decay of the solutions of 2.16 .

\section{Theorem 3.1}

Consider (3.16), where the operators $A, B(t)$ and the function $f(t)$ are defined in (3.13), (3.14) and (3.15) respectively. Then for all $z_{0} \in D(A)$ the solution $2(t)$ of (3.16) decays exponentially to 0 .

Proof

By taking norms in (3.19) and using (3.18), we get

$$
\begin{aligned}
\|z(t)\| \leqslant & M e^{-\delta t}\left\|z_{0}\right\|+\int_{0}^{t} M e^{-\delta(t-s)}\|B(s)\|\|z(s)\| d s \\
& +\int_{0}^{t} M e^{-\delta(t-s)}\|f(s)\| d s
\end{aligned}
$$

But, since $\omega(t)$ and $\dot{\omega}(t)$ are exponentially decaying, it follows from (3.14) and (3.15) that there exist positive constants $c_{1}>0, c_{2}>0, \delta_{1}>0, \delta_{2}>0$, such that for all $t \geqslant 0$

$$
\begin{gathered}
\|B(t)\| \leqslant c_{1} e^{-\delta_{1} t} \\
\|f(t)\| \leqslant c_{2} e^{-\delta_{2} t}
\end{gathered}
$$

Using (3.21) and (3.22) in (3.20), evaluating the last integral, and multiplying each side 
of $(3.20)$ by $e^{\delta t}$, we get

$$
\begin{aligned}
\left\|z(t) e^{\delta t}\right\| \leqslant & M\left\|z_{0}\right\|+\frac{M c_{2}}{\delta-\delta_{2}}\left(e^{\left(\delta-\delta_{2}\right) t}-1\right) \\
& +\int_{0}^{t} M c_{1} e^{-\delta_{1} s}\left\|z(s) e^{\delta s}\right\| d s
\end{aligned}
$$

Now applying a general form of the Bellmann-Gronwall lemma (see e.g. Desoer 1970), and using the simple estimate

$$
\int_{0}^{1} e^{-\delta_{1} s} d s \leqslant \int_{0}^{\infty} e^{-\delta_{1} s} d s \leqslant \frac{1}{\delta_{1}}
$$

we obtain the following:

$$
\begin{aligned}
\left\|z(t) e^{\delta t}\right\| \leqslant & M\left\|z_{0}\right\|+\frac{M c_{2}}{\delta-\delta_{2}}\left(e^{\left(\delta-\delta_{2}\right) t}-1\right) \\
& +\int_{0}^{t} M c_{1} e^{M c_{1} / \delta_{1}}\left[M\left\|z_{0}\right\|+\frac{M c_{2}}{\delta-\delta_{2}}\left(e^{\left(\delta-\delta_{2}\right) s}-1\right)\right] e^{-\delta_{1} s} d s \\
\leqslant & M\left\|z_{0}\right\|+\frac{M c_{2}}{\delta-\delta_{2}}\left(e^{\left(\delta-\delta_{2}\right) t}-1\right)+\frac{M^{2} c_{1}}{\delta_{1}} e^{M c_{1} / \delta_{1}}\left(\left\|z_{0}\right\|-\frac{c_{2}}{\delta-\delta_{2}}\right)\left(1-e^{-\delta_{1} t}\right) \\
& -\frac{M^{2} c_{1} c_{2}}{\left(\delta-\delta_{2}\right)\left(\delta-\delta_{1}-\delta_{2}\right)} e^{M c_{1} / \delta_{1}}\left(1-e^{\left(\delta-\delta_{1}-\delta_{2}\right) t}\right) .
\end{aligned}
$$

Multiplying each side by $e^{-\delta t}$, we obtain the desired result.

\section{Stability results for the natural control scheme}

To prove the stability of the system given by $(2.1)-(2.12)$ and $(2.13 b)$, we first define the energy of the system as

$$
\begin{aligned}
E(t)= & \frac{1}{2}\left\langle\boldsymbol{\omega}, I_{\mathbf{R}} \cdot \boldsymbol{\omega}\right\rangle+\frac{1}{2} \int_{0}^{L} \lambda\left\langle\mathbf{r}_{t}, \mathbf{r}_{t}\right\rangle d x \\
& +\frac{1}{2} \int_{0}^{L}\left(E I_{1} u_{1 x x}^{2}+E I_{3} u_{3 x x}^{2}\right) d x
\end{aligned}
$$

where $\langle$,$\rangle denotes the standard inner product in \mathbb{R}^{3}$; the first term in (4.1) is the rotational kinetic energy of the rigid body, the second term is the kinetic energy of the beam, both with respect to the inertial frame $\mathbf{N}$, and the last term is the potential energy of the beam.

\section{Proposition 4.1}

Consider the system given by $(2.1)-(2.12)$ and $(2.13 b)$. Then the energy $E(t)$ defined by (4.1) is a non-increasing function of $t$, along the solutions of $(2.1)-(2.12)$ and $(2.13 b)$.

Proof

By differentiating $E(t)$ with respect to $t$, using (2.1) and (2.4), we get 


$$
\begin{aligned}
\frac{d}{d t} E(t)= & \left\langle\boldsymbol{\omega}, \mathbf{I}_{\mathbf{R}} \cdot \dot{\boldsymbol{\omega}}\right\rangle+\int_{0}^{L} \lambda\left\langle\mathbf{r}_{t}, \mathbf{r}_{t t}\right\rangle d x \\
& +\int_{0}^{L}\left(E I_{1} u_{1 x x} u_{1 x x t}+E I_{3} u_{3 x x} u_{3 x x t}\right) d x \\
= & \left\langle\omega, \mathbf{I}_{\mathbf{R}} \cdot \dot{\omega}\right\rangle+\int_{0}^{L}\left\langle\mathbf{r}_{t}, \mathbf{n}_{x}\right\rangle d x+\int_{0}^{L}\left(E I_{1} u_{1 x x} u_{1 x x t}+E I_{3} u_{3 x x} u_{3 x x t}\right) d x \\
= & \left\langle\omega, \mathbf{I}_{\mathbf{R}} \cdot \dot{\omega}\right\rangle+\int_{0}^{L}\left\langle\omega \times \mathbf{n}_{x}\right\rangle d x-E I_{1} \int_{0}^{L} u_{1 t} u_{1 x x x x} d x-E I_{3} \int_{0}^{L} u_{3 t} u_{3 x x x x} d x \\
& +E I_{1} \int_{0}^{L} u_{1 x x} u_{1 x x t} d x+E I_{3} \int_{0}^{L} u_{3 x x} u_{3 x x t} d x
\end{aligned}
$$

Integrating by parts, we obtain the following equation, for $i=1,3$ :

$$
\begin{aligned}
E I_{i} \int_{0}^{L} u_{i t} u_{i x x x x} d x= & E I_{i} u_{i x x x}(L, t) u_{i t}(L, T)-E I_{i} u_{i x x}(L, t) u_{i x t}(L, t) \\
& +E I_{i} \int_{0}^{L} u_{i x x} u_{i x x t} d x
\end{aligned}
$$

Using (4.3) and the boundary conditions (2.11) and (2.12) in (4.2), we get

$$
\begin{aligned}
\dot{E}(t)= & -\langle\boldsymbol{\omega}, \mathbf{K} \cdot \boldsymbol{\omega}\rangle-\alpha_{1} u_{1 t}^{2}(L, t)-\alpha_{3} u_{3 t}^{2}(L, t) \\
& -\beta_{1} u_{1 x t}^{2}(L, t)-\beta_{3} u_{3 x t}^{2}(L, t) \leqslant 0
\end{aligned}
$$

Since the rate of change of the energy is non-positive, it follows that the energy is a non-increasing function of time for all $z \in H$.

\section{Remark 4.1}

If one sets $\alpha_{i}=\beta_{i}=0$, for $i=1,3$, and $\mathbf{K}=0$ (i.e. no control applied to the system), one gets $\dot{E}(t)=0$ : as expected, the total energy (given by (4.1)) is conserved.

\section{Remark 4.2}

We need an estimate that states that if the energy given by (4.1) stays bounded, then so do the beam deflections $u_{i}(x, t)$ and their derivatives $u_{i x}(x, t)$ (hence so also does $\mathbf{r}(x, t)$ ) for all $x \in[0, L]$ and for $i=1,3$. Using the boundary conditions and the fundamental theorem of calculus, for $i=1,3$ we get for all $0 \leqslant x \leqslant L$ and for all $t \geqslant 0$

$$
u_{i}(x, t)=\int_{0}^{x} u_{i s}(s, t) d s
$$

Therefore, using Jensen's inequality (see e.g. Mitrinovic 1970), we get

$$
u_{i}^{2}(x, t) \leqslant L \int_{0}^{L} u_{i s}^{2}(s, t) d s
$$

By using the same arguments, we get for all $x \in[0, L]$

$$
u_{i x}^{2}(x, t) \leqslant L \int_{0}^{L} u_{i s s}^{2}(s, t) d s
$$


Hence, combining (4.5) and (4.6), we get

$$
u_{i}^{2}(x, t) \leqslant L \int_{0}^{L} u_{i s}^{2}(s, t) d s \leqslant L^{2} \int_{0}^{L} u_{i s s}^{2}(s, t) d s
$$

Next we show that the rate of decay of the energy is at least $1 / t$ for large $t$.

\section{Theorem 4.1}

Consider the system described by $(2.1)-(2.12)$ and $(2.13 b)$. Then there exists a $T \geqslant 0$ such that the energy given by (4.1) is bounded above by $O(1 / t)$ for all $t \geqslant T$.

\section{Proof}

To show that $E(t)$ decreases at least as $O(1 / t)$, we first define the following function for any $\varepsilon \in(0,1)$ :

$$
V(t)=2(1-\varepsilon) t E(t)+2 \int_{0}^{L} i x\left\langle\mathbf{r}_{t}, \mathbf{r}_{x}\right\rangle d x
$$

Next, we need a bound on $V(t)$. Note that

$$
-\int_{0}^{L} \lambda x\left\langle\mathbf{r}_{t}, \mathbf{r}_{x}\right\rangle d x \leqslant \lambda L \int_{0}^{L}\left(\left\langle\mathbf{r}_{x}, \mathbf{r}_{x}\right\rangle+\left\langle\mathbf{r}_{t}, \mathbf{r}_{t}\right\rangle\right) d x
$$

Now by using Remark 4.2, we can find an $M_{1}>0$ and an $M_{2}>0$ such that

$$
-2 \int_{0}^{L} \lambda x\left\langle\mathbf{r}_{t}, \mathbf{r}_{x}\right\rangle d x \leqslant M_{1} E(t)+M_{2}
$$

Therefore, using this last inequality in (4.9), we get

$$
\left[2(1-\varepsilon) t-M_{1}\right] E(t)-M_{2} \leqslant V(t), \quad t \geqslant 0
$$

Now, differentiating (4.9) and using (2.1)-(2.12), we get

$$
\begin{aligned}
\dot{V}(t)= & 2(1-\varepsilon) E(t)+2(1-\varepsilon) t \dot{E}(t)+2 \int_{0}^{L} \lambda x\left\langle\mathbf{r}_{t t}, \mathbf{r}_{x}\right\rangle d x \\
& +2 \int_{0}^{L} \lambda x\left\langle\mathbf{r}_{t}, \mathbf{r}_{t x}\right\rangle d x
\end{aligned}
$$

Integrating by parts, the third and fourth integrals in (4.13) can be evaluated as follows:

$$
\begin{aligned}
2 \int_{0}^{L} \lambda x\left\langle\mathbf{r}_{t}, \mathbf{r}_{t x}\right\rangle d x & =\lambda L\left\langle\mathbf{r}_{t}(L, t), \mathbf{r}_{t}(L, t)\right\rangle-\int_{0}^{L} \lambda\left\langle\mathbf{r}_{t}, \mathbf{r}_{t}\right\rangle d x \\
\int_{0}^{L} \lambda x\left\langle\mathbf{r}_{t t}, \mathbf{r}_{x}\right\rangle d x & =\int_{0}^{L} x\left\langle\mathbf{n}_{x}, \mathbf{r}_{x}\right\rangle d x \\
& =-\int_{0}^{L} x u_{1 x} E I_{1} u_{1 x x x x} d x-\int_{0}^{L} x u_{3 x} E I_{3} u_{3 x x x x} d x
\end{aligned}
$$


To evaluate the last two integrals, we need the following:

$$
\begin{aligned}
\int_{0}^{L} x u_{x} u_{x x x x} d x= & L u_{x}(L, t) u_{x x x}(L, t)-u_{x}(L, t) u_{x x}(L, t) \\
& -\frac{1}{2} L u_{x x}^{2}(L, t)+\frac{3}{2} \int_{0}^{L} u_{x x}^{2} d x
\end{aligned}
$$

After using (4.14)-(4.16) in (4.13), we get

$$
\begin{aligned}
\dot{V}(t)= & (1-\varepsilon)\left\langle\boldsymbol{\omega}, I_{R} \boldsymbol{\omega}\right\rangle+(1-\varepsilon) \int_{0}^{L} \lambda\left\langle\mathbf{r}_{t}, \mathbf{r}_{t}\right\rangle d x \\
& +(1-\varepsilon) \int_{0}^{L}\left(E I_{1} u_{1 x x}^{2}+E I_{3} u_{3 x x}^{2}\right) d x-2(1-\varepsilon) t\langle\boldsymbol{\omega}, \mathbf{K} \cdot \boldsymbol{\omega}\rangle \\
& -2(1-\varepsilon) \alpha_{1} t u_{1 t}^{2}(L, t)-2(1-\varepsilon) \alpha_{3} t u_{3 t}^{2}(L, t) \\
& -2(1-\varepsilon) \beta_{1} t u_{1 x t}^{2}(L, t)-2(1-\varepsilon) \beta_{3} t u_{3 x t}^{2}(L, t) \\
& +\lambda L\left\langle\mathbf{r}_{t}(L, t), \mathbf{r}_{t}(L, t)\right\rangle-\int_{0}^{L} \lambda\left\langle\mathbf{r}_{t}, \mathbf{r}_{t}\right\rangle d x \\
& -2 L u_{1 x}(L, t) \alpha_{1} u_{1 t}(L, t)-2 u_{1 x}(L, t) \beta_{1} u_{1 x t}(L, t) \\
& +L \frac{\beta_{1}^{2}}{E I_{1}} u_{1 x t}^{2}(L, t)-3 E I_{1} \int_{0}^{L} u_{1 x x}^{2} d x \\
& -2 L u_{3 x}(L, t) \alpha_{3} u_{3 t}(L, t)-2 u_{3 x}(L, t) \beta_{3} u_{3 x t}(L, t) \\
& +L \frac{\beta_{3}^{2}}{E I_{3}} u_{3 x t}^{2}(L, t)-3 E I_{3} \int_{0}^{L} u_{3 x x}^{2} d x
\end{aligned}
$$

To estimate some of the terms in (4.17), we need the following inequalities:

$$
\begin{aligned}
(a+b)^{2} & \leqslant 2\left(a^{2}+b^{2}\right), \quad \alpha \in \mathbb{R}, \quad b \in \mathbb{R} \\
a b & \leqslant \delta^{2} \alpha^{2}+\frac{1}{\delta^{2}} b_{2}, \quad \delta \in \mathbb{R}, \quad \delta \neq 0, \quad a \in \mathbb{R}, \quad b \in \mathbb{R}
\end{aligned}
$$

Finally, using Proposition 4.1 and Remark 4.2, we get the following estimate of the end-point velocities of the beam in the inertial frame:

$$
\left\langle\mathbf{r}_{t}(L, t), \mathbf{r}_{t}(L, t)\right\rangle \leqslant k_{1}\left[u_{1 t}^{2}(L, t)+u_{3 t}^{2}(L, t)\right]+k_{2}\langle\boldsymbol{\omega}, \boldsymbol{\omega}\rangle
$$

for some $k_{1}>0$ and $k_{2}>0$. Using these estimates in (4.17), we obtain

$$
\begin{aligned}
\dot{V}(t) \leqslant & -(1-\varepsilon)\left(2 t\langle\boldsymbol{\omega}, \mathbf{K} \cdot \boldsymbol{\omega}\rangle-\left\langle\boldsymbol{\omega}, \mathbf{I}_{\mathbf{R}} \cdot \boldsymbol{\omega}\right\rangle-\lambda L k_{2}\langle\boldsymbol{\omega}, \boldsymbol{\omega}\rangle\right) \\
& -\varepsilon \int_{0}^{L} \hat{\lambda}\left\langle\mathbf{r}_{t}, \mathbf{r}_{t}\right\rangle d x-(\varepsilon+2) \int_{0}^{L}\left(E I_{1} u_{1 x x}^{2}+E I_{3} u_{3 x x}^{2}\right) d x \\
& -2(1-\varepsilon) \alpha_{1} t u_{1 t}^{2}(L, t)-2(1-\varepsilon) \alpha_{3} t u_{3 t}^{2}(L, t) \\
& -2(1-\varepsilon) \beta_{1} t u_{1 x t}^{2}(L, t)-2(1-\varepsilon) \beta_{3} t u_{3 x t}^{2}(L, t) \\
& +\lambda L k_{1}\left[u_{1 t}^{2}(L, t)+u_{3 t}^{2}(L, t)\right]+2 L \alpha_{1}\left[\delta_{1}^{2} u_{1 x}^{2}(L, t)+\frac{1}{\delta_{1}^{2}} u_{1 t}^{2}(L, t)\right]
\end{aligned}
$$




$$
\begin{aligned}
& +2 L \alpha_{3}\left[\delta_{2}^{2} u_{3 x}^{2}(L, t)+\frac{1}{\delta_{2}^{2}} u_{3 t}^{2}(L, t)\right]+\delta_{3}^{2} u_{1 x}^{2}(L, t) \\
& +2 \beta_{1}\left[\delta_{3}^{2} u_{1 x}^{2}(L, t)+\frac{1}{\delta_{3}^{2}} u_{1 t}^{2}(L, t)\right] \\
& +2 \beta_{3}\left[\delta_{4}^{2} u_{3 x}^{2}(L, t)+\frac{1}{\delta_{4}^{2}} u_{3 x t}^{2}(L, t)\right] \\
& +\frac{L}{E I_{1}} \beta_{1}^{2} u_{1 x t}^{2}(L, t)+\frac{L}{E I_{3}} \beta_{3}^{2} u_{3 x t}^{2}(L, t)
\end{aligned}
$$

where $\delta_{i} \in \mathbb{R}$ are any non-zero real numbers, for $i=1, \ldots, 4$. Now, collecting like terms, we rearrange (4.21) as follows:

$$
\begin{aligned}
\dot{V}(t) \leqslant & -(1-\varepsilon)\left(2 t\langle\boldsymbol{\omega}, \mathbf{K} \cdot \boldsymbol{\omega}\rangle-\left\langle\boldsymbol{\omega}, \mathbf{I}_{R} \cdot \boldsymbol{\omega}\right\rangle-\lambda L k_{2}\langle\boldsymbol{\omega}, \boldsymbol{\omega}\rangle\right) \\
& -\varepsilon \int_{0}^{L} \lambda\left\langle\mathbf{r}_{t}, \mathbf{r}_{t}\right\rangle d x-\left[2(1-\varepsilon) \alpha_{1} t-\lambda L k_{1}-\frac{2 L \alpha_{1}}{\delta_{1}^{2}}\right] u_{1 t}^{2}(L, t) \\
& -\left[2(1-\varepsilon) \alpha_{3} t-\lambda L k_{1}-\frac{2 L \alpha_{3}}{\delta_{2}^{2}}\right] u_{3 t}^{2}(L, t) \\
& -\left[2(1-\varepsilon) \beta_{1} t-\frac{2 \beta_{1}}{\delta_{3}^{2}}-L \frac{\beta_{1}^{2}}{E I_{1}}\right] u_{1 x t}^{2}(L, t) \\
& -\left[2(1-\varepsilon) \beta_{3} t-2 \frac{\beta_{3}}{\delta_{4}^{2}}-L \frac{\beta_{3}^{2}}{E I_{3}}\right] u_{3 x t}^{2}(L, t) \\
& -(\varepsilon+2) \cdot \int_{0}^{L}\left(E I_{1} u_{1 x x}^{2}+E I_{3} u_{3 x x}^{2}\right) d x \\
& +\left(2 L \alpha_{1} \delta_{1}^{2}+2 \beta_{1} \delta_{3}^{2}\right) u_{1 x}^{2}(L, t)+\left(2 L \alpha_{3} \delta_{2}^{2}+2 \beta_{3} \delta_{4}^{2}\right) u_{3 x}^{2}(L, t)
\end{aligned}
$$

By Remark 4.2 (e.g. using (4.7)) and choosing $\delta_{i}$ sufficiently small, $i=1, \ldots, 4$, the sum of the last two lines in (4.22) can be made negative. We then conclude that after some $T \in \mathbb{R}$

$$
\dot{V}(t) \leqslant 0 \quad(t \geqslant T)
$$

hence

$$
V(t) \leqslant V(T) \quad(t \geqslant T)
$$

Using (4.12) and (4.24), we get the following estimate, which proves Theorem 4.1.

$$
E(t) \leqslant \frac{V(T)+M_{2}}{2(1-\varepsilon) t-M_{1}} \quad(t \geqslant T)
$$

For the sake of clarity, the existence, uniqueness and exponential decay of the solutions of the equations given by $(2.1)-(2.12)$ and $(2.13 b)$ are presented in the next section.

\section{Existence, uniqueness and exponential decay of solutions}

In this section we first give an existence and uniqueness theorem for the linear part of the equations (2.1)-(2.12) and $(2.13 b)$ (i.e. the 'natural' control scheme). Then, 
including the non-linear terms in Theorem 5.2 we prove the exponential decay of the solutions of the same equations.

For simplicity, we take the positive-definite matrix $\mathbf{K}=\operatorname{diag}\left(k_{1}, k_{2}, k_{3}\right)$. Then $(2.1)-(2.12)$ and $(2.13 b)$ can be written as

$$
\begin{aligned}
& E I_{1} u_{1 x x x x}+\lambda u_{1 t t}+2 \lambda \omega_{2} u_{3 t}+\lambda\left(\dot{\omega}_{2}+\omega_{1} \omega_{3}\right) u_{3} \\
& \quad-\lambda\left(\omega_{2}^{2}+\omega_{3}^{2}\right) u_{1}-\lambda\left(\dot{\omega}_{3}-\omega_{1} \omega_{2}\right)(b+x)=0, \quad 0<x<L, \quad t \geqslant 0 \\
& \begin{aligned}
E I_{3} u_{3 x x x x} & +\lambda u_{3 t t}-2 \lambda \omega_{2} u_{1 t}-\lambda\left(\dot{\omega}_{2}-\omega_{1} \omega_{3}\right) u_{1} \\
& -\lambda\left(\omega_{1}^{2}+\omega_{2}^{2}\right) u_{3}+\lambda\left(\dot{\omega}_{1}+\omega_{2} \omega_{3}\right)(b+x)=0, \quad 0<x<L, \quad t \geqslant 0
\end{aligned} \\
& I_{1} \dot{\omega}_{1}+\left(I_{3}-I_{2}\right) \omega_{2} \omega_{3}+k_{1} \omega_{1}=E I_{2} \int_{0}^{L}(b+x) u_{3 x x x x} d x \\
& I_{2} \dot{\omega}_{2}+\left(I_{1}-I_{3}\right) \omega_{3} \omega_{1}+k_{2} \omega_{2}=E I_{3} \int_{0}^{L} u_{1} u_{3 x x x x} d x-E I_{1} \int_{0}^{L} u_{3} u_{1 x x x x} d x \\
& I_{3} \dot{\omega}_{3}+\left(I_{2}-I_{1}\right) \omega_{1} \omega_{2}+k_{3} \omega_{3}=E I_{1} \int_{0}^{L}-(b+x) u_{1 x x x x} d x
\end{aligned}
$$

together with the boundary conditions (2.9), (2.11) and (2.12). Let the function space $H$ be the same as defined in (3.11). Define a new function space $\hat{H}=H \times \mathbb{R}^{3}$. Then, separating the linear and non-linear parts, (5.1)-(5.5) can be put into the following matrix form:

$$
\frac{d z}{d t}=\hat{A} z+T_{1}(z)+g(z)
$$

where $z=\left[\begin{array}{lllllll}u_{1} & u_{1 t} & u_{3} & u_{3 t} & \omega_{1} & \omega_{2} & \omega_{3}\end{array}\right]^{\mathrm{T}} . \hat{A}: \hat{H} \rightarrow \hat{H}$ is a linear operator whose matrix form is specified by

$$
\hat{A}=\left\{m_{i j}: i=1, \ldots, 7, j=1, \ldots, 7\right\}
$$

where all $m_{i j}$ are zero except

$$
\begin{aligned}
& m_{12}=m_{34}=1 \\
& m_{21}=-\frac{E I_{1}}{\lambda} \frac{\partial^{4}}{\partial x^{4}}-\frac{E I_{1}}{I_{3}}(b+x) \int_{0}^{L}(b+x) \frac{\partial^{4}}{\partial x^{4}} d x, \quad m_{27}=-\frac{k_{3}}{I_{3}}(b+x) \\
& m_{43}=-\frac{E I_{3}}{\lambda} \frac{\partial^{4}}{\partial x^{4}}-\frac{E I_{3}}{I_{1}}(b+x) \int_{0}^{L}(b+x) \frac{\partial 4}{\partial x^{4}} d x, \quad m_{45}=\frac{k_{1}}{I_{1}}(b+x) \\
& m_{53}=\frac{E I_{3}}{I_{1}} \int_{0}^{L}(b+x) \frac{\partial^{4}}{\partial x^{4}} d x, \quad m_{55}=-\frac{k_{1}}{I_{1}}, \quad m_{66}=-\frac{k_{2}}{I_{2}} \\
& m_{71}=-\frac{E I_{1}}{I_{3}} \int_{0}^{L}(b+x) \frac{\partial^{4}}{\partial x^{4}} d x, \quad m_{77}=-\frac{k_{3}}{I_{3}}
\end{aligned}
$$


$T_{1}: \hat{H} \rightarrow \hat{H}$ is a non-linear integral operator defined by

$$
T_{1}(z)=\left[\begin{array}{c}
0 \\
u_{3} \int_{0}^{L}\left(-\frac{E I_{1}}{I_{2}} u_{3} u_{1 x x x x}+\frac{E I_{3}}{I_{2}} u_{1} u_{3 x x x x}\right) d x \\
0 \\
u_{1} \int_{0}^{L}\left(-\frac{E I_{3}}{I_{2}} u_{1} u_{3 x x x x}+\frac{E I_{1}}{I_{2}} u_{3} u_{1 x x x x}\right) d x \\
0 \\
\int_{0}^{L}\left(-\frac{E I_{3}}{I_{2}} u_{1} u_{3 x x x x}+\frac{E I_{1}}{I_{2}} u_{3} u_{1 x x x x}\right) d x \\
0
\end{array}\right]
$$

$g: \hat{H} \rightarrow \hat{H}$ is a non-linear operator given by

$$
g(z)=\left[\begin{array}{lll}
g_{1}(z) & \ldots & g_{7}(z)
\end{array}\right]^{\mathrm{T}}
$$

where the $g_{i}(z)$ are defined by

$$
\begin{aligned}
g_{1}(z)= & g_{3}(z)=0 \\
g_{2}(z)= & \frac{I_{1}-I_{3}}{I_{2}} \omega_{1} \omega_{3} u_{3}+\frac{I_{1}-I_{2}}{I_{3}} \omega_{1} \omega_{2}(b+x) \\
& +\frac{k_{2}}{I_{2}} \omega_{2} u_{3}-2 \omega_{2} u_{3 t}+\left(\omega_{2}^{2}+\omega_{3}^{2}\right) u_{1}-\omega_{1} \omega_{3} u_{3}-\omega_{1} \omega_{2}(b+x) \\
g_{4}(z)= & \frac{I_{3}-I_{1}}{I_{2}} \omega_{1} \omega_{3} u_{1}-\frac{I_{2}-I_{3}}{I_{1}} \omega_{2} \omega_{3}(b+x) \\
& -\frac{k_{2}}{I_{2}} \omega_{2} u_{1}+2 \omega_{2} u_{1 t}+\left(\omega_{1}^{2}+\omega_{2}^{2}\right) u_{3}-\omega_{1} \omega_{3} u_{1}-\omega_{2} \omega_{3}(b+x) \\
g_{5}(z)= & \frac{I_{2}-I_{3}}{I_{1}} \omega_{2} \omega_{3}, \quad g_{6}(z)=\frac{I_{3}-I_{1}}{I_{2}} \omega_{1} \omega_{3}, \quad g_{7}(z)=\frac{I_{1}-I_{2}}{I_{3}} \omega_{1} \omega_{2}
\end{aligned}
$$

Note that $\hat{A}: \hat{H} \rightarrow \hat{H}$ is an unbounded linear operator and its domain $D(\hat{A})$ is defined as $D(\hat{A})=D(A) \times \mathbb{R}^{3}$, where $D(A)$ is defined in (3.17) and is dense in $\hat{H}$, since $D(A)$ is dense in $H$.

In $\hat{H}$ we define the following 'energy' inner product:

$$
\begin{aligned}
\langle z, \hat{z}\rangle_{1}= & I_{1} \omega_{1} \hat{\omega}_{1}+I_{2} \omega_{2} \hat{\omega}_{2}+I_{3} \omega_{3} \hat{\omega}_{3} \\
& +\int_{0}^{L} \lambda\left[u_{1 t}-\omega_{3}(b+x)\right]\left[\hat{u}_{1 t}-\hat{\omega}_{3}(b+x)\right] d x \\
& +\int_{0}^{L} \lambda\left[u_{3 t}+\omega_{1}(b+x)\right]\left[\hat{u}_{3 t}+\hat{\omega}_{1}(b+x)\right] d x \\
& +\int_{0}^{L}\left(E I_{1} u_{1 x x} \hat{u}_{1 x x}+E I_{3} u_{3 x x} \hat{u}_{3 x x}\right) d x
\end{aligned}
$$


This inner product induces a norm on $\hat{H}$ :

$$
\begin{aligned}
\|z\|_{1}^{2}= & 2 \hat{E}(t)=I_{1} \omega_{1}^{2}+I_{2} \omega_{2}^{2}+I_{3} \omega_{3}^{2} \\
& +\int_{0}^{L} \lambda\left\{\left[u_{1 t}-\omega_{3}(b+x)\right]^{2}+\left[u_{3 t}+\omega_{1}(b+x)\right]^{2}\right\} d x \\
& +\int_{0}^{L}\left(E I_{1} u_{1 x x}^{2}+E I_{3} u_{3 x x}^{2}\right) d x
\end{aligned}
$$

Note that the usual 'Sobolev' type norm that makes $\hat{H}$ a Banach space is given by

$$
\begin{aligned}
\|z\|^{2}= & \omega_{1}^{2}+\omega_{2}^{2}+\omega_{3}^{2}+\int_{0}^{L}\left(u_{1}^{2}+u_{1 x}^{2}+u_{1 x x}^{2}\right) d x \\
& +\int_{0}^{L}\left(u_{3}^{2}+u_{3 x}^{2}+u_{3 x x}^{2}\right) d x+\int_{0}^{L}\left(u_{1 t}^{2}+u_{3 t}^{2}\right) d x
\end{aligned}
$$

But by Remark 4.2 and inequalities (4.18)-(4.19) it can be shown that the norms given by (5.11) and (5.10) are equivalent to each other.

\section{Theorem 5.1}

Consider the linear operator $\hat{A}: \hat{H} \rightarrow \hat{H}$ given by (5.7). Then

(i) $\hat{A}$ generates a $C_{0}$ semigroup $\hat{T}(t)$;

(ii) there exist positive constants $M>0$ and $\delta>0$ such that

$$
\|\hat{T}(t)\| \leqslant M e^{-\delta t} \quad(t \geqslant 0)
$$

Proof

(i) We shall use the Lumer-Phillips theorem to prove (i) (see Pazy 1983, p. 14). So we have to show that $\hat{A}$ is dissipative and the operator $(\lambda I-\hat{A}): \hat{H} \rightarrow \hat{H}$ is onto for some $\lambda>0$.

To prove that $\hat{A}$ is dissipative, consider the equation

$$
\frac{d z}{d t}=\hat{A} z, \quad z(0) \in D(\hat{A})
$$

Then, differentiating (5.10) and using (5.13) and (5.7), we get

$$
\begin{aligned}
\frac{d \hat{E}}{d t}= & I_{1} \omega_{1} \dot{\omega}_{1}+I_{2} \omega_{2} \dot{\omega}_{2}+I_{3} \omega_{3} \dot{\omega}_{3}+\int_{0}^{L} \lambda\left[u_{1 t}-\omega_{3}(b+x)\right]\left[u_{1 t t}-\dot{\omega}_{3}(b+x)\right] d x \\
& +\int_{0}^{L} \lambda\left[u_{3 t}+\omega_{1}(b+x)\right]\left[u_{3 t t}+\dot{\omega}_{1}(b+x)\right] d x \\
& +\int_{0}^{L}\left(E I_{1} u_{1 x x} u_{1 x x t}+E I_{3} u_{3 x x} u_{3 x x t}\right) d x \\
= & -k_{1} \omega_{1}^{2}-k_{2} \omega_{2}^{2}-k_{3} \omega_{3}^{2}-\alpha_{1} u_{1 t}^{2}(L, t) \\
& -\alpha_{3} u_{3 t}^{2}(L, t)-\beta_{1} u_{1 x t}^{2}(L, t)-\beta_{3} u_{3 x t}^{2}(L, t) \leqslant 0
\end{aligned}
$$

This proves that $\hat{A}$ is dissipative.

To prove that the linear operator $(\lambda I-\hat{A}): \hat{H} \rightarrow \hat{H}$ is onto for some $\lambda>0$, we 
decompose $\hat{A}$ as follows:

$$
\hat{A}=A_{1}+T_{\mathrm{D}}
$$

where $A_{1}: \hat{H} \rightarrow \hat{H}$ is defined as

$$
A_{1}=\left[\begin{array}{ccccccc}
0 & 1 & 0 & 0 & 0 & 0 & 0 \\
-\frac{E I_{1}}{\lambda} \frac{\partial 4}{\partial x^{4}} & 0 & 0 & 0 & 0 & 0 & 0 \\
0 & 0 & 0 & 1 & 0 & 0 & 0 \\
0 & 0 & -\frac{E I_{3}}{\lambda} \frac{\partial^{4}}{\partial x^{4}} & 0 & 0 & 0 & 0 \\
0 & 0 & 0 & 0 & -\frac{k_{1}}{I_{1}} & 0 & 0 \\
0 & 0 & 0 & 0 & 0 & -\frac{k_{2}}{I_{2}} & 0 \\
0 & 0 & 0 & 0 & 0 & 0 & -\frac{k_{3}}{I_{3}}
\end{array}\right]
$$

and the operator $T_{\mathrm{D}}: \hat{H} \rightarrow \hat{H}$ is defined as

$$
T_{\mathrm{D}}=\hat{A}-A_{1}
$$

We first note the following.

(1) $A_{1}: \hat{H} \rightarrow \hat{H}$ is a linear unbounded operator. Its domain $D\left(A_{1}\right)$ is equal to $D(\hat{A})$. By using Theorem 3.1 of Chen (1987), it can be shown that $A_{1}$ generates an $C_{0}$ contraction semigroup. Hence $\left(\lambda I-A_{1}\right): \hat{H} \rightarrow \hat{H}$ is an invertible operator for all $\lambda>0$. In fact the range of $\left(\lambda I-A_{1}\right)^{-1}$ is equal to $D\left(A_{1}\right)$ and by the Hille-Yosida theorem (see e.g. Pazy 1983, p. 8) we have

$$
\left\|\left(\lambda I-A_{1}\right)^{-1}\right\| \leqslant \frac{1}{\lambda}, \quad \lambda>0, \quad \lambda \in \mathbb{R}
$$

(2) $T_{\mathrm{D}}: \hat{H} \rightarrow \hat{H}$ is a degenerate linear operator relative to the $A_{1}$ (see Kato 1980 , p. 245). By definition, the range space. of $T_{\mathrm{D}}$ is finite-dimensional and there exist positive constants $a$ and $b$ such that

$$
\left\|T_{\mathrm{D}} z\right\| \leqslant a\|z\|+b\left\|A_{1} z\right\| \quad \text { for all } z \in D\left(A_{1}\right)
$$

That the operator $T_{\mathrm{D}}$ has a finite dimensional range follows from (5.17), (5.7) and (5.16).

By using (5.17) and (5.11), it can be shown that (5.18) holds for some positive $a$ and $b$.

From the remarks (1) and (2) above, it follows that $T_{\mathrm{D}}\left(\lambda I-A_{1}\right)^{-1}: \hat{H} \rightarrow \hat{H}$ is a bounded linear operator with finite-dimensional range; hence $\left\|T_{\mathrm{D}}\left(\lambda I-A_{1}\right)^{-1}\right\| \leqslant M$ for some $M>0$, and $T_{\mathrm{D}}\left(\lambda I-A_{1}\right)^{-1}$ is a compact operator (see Kato 1980, p. 245).

Next we need the following fact. 
Fact 5.1

For all $\lambda>0,1$ is not an eigenvalue of the compact operator $T_{\mathrm{D}}\left(\lambda I-A_{1}\right)^{-1}$.

Proof

Suppose not. Then there exists a $\lambda>0$ and a $y \in \hat{H}, y \neq 0$, such that

$$
y=T_{\mathrm{D}}\left(\lambda I-A_{1}\right)^{-1} y
$$

Define $x \in D\left(A_{1}\right)$ as

$$
x=\left(\lambda I-A_{1}\right)^{-1} y
$$

Then (5.19) implies that the following also holds:

$$
\left(\lambda I-A_{1}-T_{\mathrm{D}}\right) x=0
$$

But, since $\hat{A}=A_{1}+T_{\mathrm{D}}$ is dissipative and $\lambda>0$, it follows that $x=0$, which implies $y=0$, which is a contradiction

From Fact 5.1 it follows that the operator $I-T_{\mathrm{D}}\left(\lambda I-A_{1}\right)^{-1}$ is invertible for all $\lambda>0$. Hence we conclude that $\left(\lambda I-A_{1}-T_{\mathrm{D}}\right): \hat{H} \rightarrow \hat{H}$ is invertible for all $\lambda>0$ and its inverse is given by

$$
\left(\lambda I-A_{1}-T_{\mathrm{D}}\right)^{-1}=\left(\lambda I-A_{1}\right)^{-1}\left[I-T_{\mathrm{D}}\left(\lambda I-A_{1}\right)^{-1}\right]^{-1}
$$

This shows that $\left(\lambda I-A_{1}-T_{\mathrm{D}}\right): \hat{H} \rightarrow \hat{H}$ is onto for all $\lambda>0$. Then assertion (i) follows from the Lumer-Phillips theorem (see Pazy 1980).

(ii) To prove that the semigroup $\hat{T}(t)$ generated by $\hat{A}$ is exponentially decaying, we first follow a similar argument to that we made in proving Theorem 4.1. We first define the function

$$
\begin{aligned}
\hat{V}(t)= & 2(1-\varepsilon) t \hat{E}(t)+2 \int_{0}^{L} \lambda x\left[u_{1 t}-\omega_{3}(b+x)\right] u_{1 x} d x \\
& +2 \int_{0}^{L} \lambda x\left[u_{3 t}+\omega_{1}(b+x)\right] u_{3 x} d x
\end{aligned}
$$

where $\varepsilon \in(0,1)$ is arbitrary. Applying Schwartz's inequality to the integrals in $(5.20)$, it can be shown that there exists a $K>0$ such that

$$
[2(1-\varepsilon) t-K] \hat{E}(t) \leqslant \hat{V}(t)
$$

Differentiating $\hat{V}(t)$ with respect to $t$, using (5.1)-(5.5) and following the line of the proof of Theorem 4.1, we conclude that there exists a $T>0$ such that $\hat{V}(t)$ is bounded above for all $t \geqslant T$. Therefore $\hat{E}(t)$ is bounded above by $O(1 / t)$ for all $t \geqslant T$. Hence for some $M>0$

$$
\int_{0}^{\infty} \hat{E}^{2}(t) d t \leqslant M
$$

Assertion (ii) then follows from a theorem due to Pazy (1980, p. 116).

We now show the existence and uniqueness of the solutions of (5.6). The main difficulty is the fact that the non-linear operator $T_{\mathrm{I}}(z): \hat{H} \rightarrow \hat{H}$ defined by (5.8) is also unbounded, that is not defined for all $z \in \hat{H}$. But, with an appropriate norm defined on $D(\hat{A})$ (see (5.21) below), $T_{1}(z): D(\hat{A}) \rightarrow \hat{H}$ becomes an $C^{\infty}$ operator. 


\section{Theorem 5.2}

Consider (5.6), where the operators $\hat{A}, T_{\mathrm{D}}$ and $g$ are defined in the (5.7)-(5.9). Then

(i) for all initial conditions $z(0) \in D(\hat{A}),(5.6)$ has a unique classical solution $z(t)$ defined for all $t>0$;

(ii) in terms of the semigroup $\hat{T}(t)$ generated by the linear operator $\hat{A}$, this solution can be written as

$$
z(t)=\hat{T}(t) z(0)+\int_{0}^{t} \hat{T}(t-s) T_{1}(z(s)) d s+\int_{0}^{t} \hat{T}(t-s) g(z(s)) d s
$$

(iii) the solutions of (5.6) are exponentially decaying.

\section{Proof}

(i) Following Segal (1963), we define the following norm on $D(\hat{A})$ :

$$
\|z\|=\left\|\hat{A}_{z}\right\|, \quad z \in D(\hat{A})
$$

where $\|\cdot\|$ is defined in (5.11). A simple calculation shows that this norm is equivalent to a standard Sobolov norm for $D(\hat{A})$; hence $D(\hat{A})$ with this norm becomes a Banach space. Let us call this space $[D(\hat{A})]$. Then $T_{1}:[D(\hat{A})] \rightarrow \hat{H}$ becomes an $C^{\infty}$ operator, since its components are linear combinations of products and integrals of the components of $z$ over $[0, L]$ (see (5.6) and (5.8)).

Note also that $g: \hat{H} \rightarrow \hat{H}$, as defined by (5.9), is a $C^{\infty}$ map, since its components are products of the components of $z$. Therefore it follows from Theorem 2 of Segal (1963) that (5.6) has a unique classical solution for all initial conditions $z(0) \in D(\hat{A})$, defined in $[0, \delta]$ for some $\delta>0$. But, since Theorem 4.1 shows that the solutions decay to 0 , this local existence theorem can be extended globally (i.e. for all $t>0$ ).

(ii) This may be proved by substitution in (5.6).

(iii) Since by Theorem 4.1 the solutions of (5.6) decay to 0 in $\hat{H}$, it follows that the positive orbits $O_{0}^{+}(t)=\left\{\left.z(t) \in \hat{H}\right|_{z(0)}(0)=z_{0}, t>0\right\}$ belong to a compact set in $\hat{H}$. Therefore, by a generalization of LaSalle's invariance argument to infinitedimensional spaces (see e.g. Hale 1969), and by the energy decay estimate (4.4) it follows that the rate of change of the energy defined by (4.4) decays asymptotically to 0 . That is, $u_{i t}(L, t), u_{i x t}(L, t), i=1,3$ and $\omega(t)$ decay to 0 as $t \rightarrow \infty$. Then integrating by parts in (5.8) and using the same techniques as in the proof of Theorem 4.1, we obtain the following bounds:

$$
\begin{aligned}
\left\|T_{1}(z(t))\right\| & \leqslant \gamma_{1}(t)\|z(t)\| \\
\|g(z(t))\| & \leqslant \gamma_{2}(t)\|z(t)\|
\end{aligned}
$$

where $\gamma_{1}(t)$ and $\gamma_{2}(t)$ decay asymptotically to 0 . From these, and following the arguments used in the proof of Theorem 3.1, we conclude that the solutions of (5.6) decay exponentially to 0 .

\section{ACKNOWLEDGMENTS}

We should like to thank Professor Charles A. Desoer for his guidance, encouragement and careful review of this manuscript, and to John Anagnost for many helpful discussions and numerous helpful comments. We should also like to thank Professor J. L. Sackman and Professor P. M. Naghdi for many helpful comments. 
This research has been partially supported by the National Science Foundation under Grant ECS 8500993 and by the Scientific and Technical Research Council of Turkey.

\section{REFERENCES}

Antman, S. S., 1972, The Theory of Rods. Handbuch der Physik, Vol. 6 a/2 (Berlin: SpringerVerlag).

Balas, M. J., 1982, Trends in large space structure control theory: fondest hopes, wildest dreams. I.E.E.E. Transactions on Automatic Control, 27, 522-535.

BaLAS, M. J., 1978, Modal control of certain flexible dynamical systems. SIAM Journal of Control, 16, 450-462.

Biswas, S. K., and Aнmed, N. U., 1986, Stabilization of a class of hybrid system arising in flexible spacecraft. Journal of Optimization Theory and Applications, 50, 83-108.

CHEN, G., 1979, Energy decay estimates and exact boundary value controllability for the wave equation in a bounded domain. Journal Mathématiques Pures et Appliquées, 58, 249-273.

Chen, G., Delfour, M. C., Krall, A. M., and Payre, G., 1987, Modeling, stabilization and control of serially connected beams. SIAM Journal of Control and Optimization, 25, $526-546$.

Desoer, C. A., 1970, Notes for a Second Course on Linear Systems (New York: Van Nostrand Reinhold).

Desoer, C. A., and Morgul, O., 1988, Control and stabilization of a flexible beam attached to a rigid body: planar motion. Proceedings of the 27th CDC, Austin. Texas, pp. 1643-1645.

Goldstein, H., 1980, Classical Mechanics, 2nd edition (Reading, Massachusetts: AddisonWesley)

HALE, J. K., 1969, Dynamical systems and stability. Journal of Mathematical Analysis and Applications, 26, 39-59.

Kane, T. R., and Levinson, D. A., 1985, Dynamics: Theory and Applications (New York: McGraw-Hill).

Kato, T., 1980, Perturbation Theory for Linear Operators, 2nd edition (Berlin: Springer-Verlag).

MarSDen, J., and Hughes, T. J. R., 1983, Mathematical Foundations of Elasticity (Englewood Cliffs, New Jersey: Prentice-Hall).

Meirovitch, L., 1967, Analytical Methods in Vibration (New York: MacMillan).

Mitrinovic, D. S., 1970, Analytical Inequalities (Berlin: Springer-Verlag).

PaUl, R. P., 1981, Robot Manipulators: Mathematics, Programming and Control (Cambridge: Massachusetts: MIT Press).

Pazy, A., 1983, Semigroups of Linear Operators and Applications to Partial Differential Equations (Berlin: Springer-Verlag).

Posbergh, T. A., 1988, Modeling and control of mixed and flexible structures. Ph.D. dissertation, Technical Research Report, Systems Research Center, University of Maryland, SRC TR 88-58.

Segal, I., 1963, Nonlinear semigroups. Annals of Mathematics, 78, 339-364. 